\title{
ESTUDIO DESCRIPTIVO DEL PAPEL DE LA TRANSFERENCIA LINGÜÍSTICA EN LA ADQUISICIÓN DE LA L2: PRINCIPALES APORTACIONES TEÓRICAS DE LA SEGUNDA MITAD DEL SIGLO XX.
}

\author{
José Enrique García González
}

\section{Conceptos y terminología}

El fenómeno de la transferencia lingüística está relacionado generalmente con las situaciones de contacto entre lenguas, ya sea en el contexto de poblaciones enteras (p. ej. el contacto lingüístico entre el inglés y el español en la comunidad de Gibraltar) o en el plano del individuo, siendo éste último el caso de la adquisición de una segunda lengua ${ }^{1}$. El presente artículo, en concreto, constituye una descripción del papel cambiante que ha desempeñado la transferencia lingüística en el ámbito de la adquisición ${ }^{2}$ de una segunda lengua (L2) ${ }^{3}$, tomando como referencia las principales aportaciones teóricas en este campo durante la segunda mitad del siglo XX.

Como punto de partida, hay que señalar que el fenómeno de la 'influencia entre lenguas', y en nuestro caso concreto de la influencia y los efectos de la L1 o lengua materna en la L2, no está exento de problemas terminológicos y conceptuales. En un principio, el término más utilizado para referirse a dicha influencia lingüística es el de interferencia (ingl. interference), que conlleva un carácter negativo derivado de su sentido etimológico: 'golpearse mutuamente', 'interponerse'4. Desde esta perspectiva, algunos autores estructuralistas, como Jakobson y Sandfeld ${ }^{5}$, señalan que la interferencia se produce cuando

\footnotetext{
${ }^{1}$ Véase Dechert, Brüggemeier y Fütterer (1984) para una compilación de bibliografía sobre el tema de la transferencia y sus distintos campos de aplicación.

${ }^{2}$ Para los objetivos del presente trabajo, y siguiendo a autores como Odlin (1989) y Ellis (1985), los términos adquisición y aprendizaje se emplean indistintamente. Krashen, en cambio, establece una distinción entre ambos conceptos: el primero se realiza de forma inconsciente y normalmente en un entorno natural, mientras que el segundo es el resultado de una instrucción o estudio consciente y tiene lugar en un entomo formal (cfr. Krashen 1981).

${ }^{3}$ Por L2 entendemos tanto segunda lengua como lengua extranjera.

${ }^{4}$ Véase J. Corominas, Breve diccionario etimológico de la lengua castellana, $3^{\mathrm{a}}$ ed. (Madrid 1990) 338.

${ }^{5} \mathrm{R}$. Jakobson, "Sur la théorie des affinités phonologiques des langues", Actes du Quatrième Congrès des Linguistes (1938) 48-58;; K. Sandfeld, "Problèmes d'interférences linguistiques", Actes du Quatrième Congrès des Linguistes (1938) 59-61.
} 
un rasgo perteneciente a un código lingüístico se introduce en otro sistema en el que resulta ajeno, causando determinadas modificaciones o "perturbaciones" en la estructura del mismo.

En el terreno de la sociolingüística, Weinreich (1953) es uno de los pioneros en el estudio de la interferencia linguiística en relación con los problemas derivados del bilingüismo y del contacto entre las lenguas. Este autor define la interferencia como "those instances of deviation [cursiva mía] from the norms of either language which occur in the speech of bilinguals as a result of the familiarity with more than one language, i.e. as a result of language contact" (Weinreich 1953:1). Pero a diferencia del enfoque estructuralista de los mencionados autores, Weinreich considera como causas de la interferencia tanto los factores estructurales o lingüísticos como los no estructurales o extralingüísticos (el mayor o menor prestigio de las lenguas que están en contacto, las actitudes hacia las culturas respectivas, etc.).

El concepto de interferencia también empieza a tener protagonismo en el terreno de la enseñanza y el aprendizaje de lenguas extranjeras. En tales casos, la noción de interferencia, muy influenciada por la corriente psicológica del behaviorismo dominante en las años cincuenta y sesenta, presenta un marcado carácter negativo y se considera la causa de los distintos errores que cometen los aprendices de lenguas (v. apdo. 2.1.).

Como apuntan las definiciones anteriores, en las diferentes ramas de la lingüística han predominado las interpretaciones de la interferencia bajo una óptica negativa y algo imprecisa. Como observa Payrató ${ }^{6}$, el término se ha asociado a otros tradicionalmente conocidos bajo las denominaciones de "injerencia", "intromisión" o "infiltración".

A la vista de estas consideraciones, algunos autores han matizado el concepto de interferencia encuadrándolo en un contexto más amplio ${ }^{7}$ o han preferido utilizar otros términos. En este sentido, Odlin (1989) opta por los vocablos transferencia (transfer) o influencia interlinguiística (cross-linguistic influence) para designar "the influence resulting from similarities and differences between the target language and any other language that has been previously (...) acquired" (pág. 27). En este contexto, distingue entre una transferencia positiva y una negativa. La primera facilita el proceso de adquisición de la L2 como resultado de la semejanza estructural entre ambas lenguas. Por el contrario, la transferencia negativa, a la que también denomina interferencia, es el producto de las diferencias entre la L1 y la L2 y se manifiesta en errores y "other effects that constitute a divergence between the behavior of native and non-native speakers of a language" (Odlin 1989:167).

Kellerman y Sharwood Smith (1986) prefieren utilizar el término influencia interlinguiística entendido como "the interplay between earlier and later acquired

\footnotetext{
${ }^{6}$ Ll. Payrató, La interferència lingüística (Comentaris i exemples català-castellà) (Barcelona 1985) 51.

${ }^{7}$ Cfr. R. Sandor, Language Contact (Frankfurt am Mein 1991) 28-29. Este autor señala que el término interferencia sigue teniendo un uso muy difundido, pero precisa que es necesario entender el fenómeno como la interacción mutua entre dos sistemas linguísticos en lugar de considerar sólo la influencia negativa de uno sobre otro.
} 
languages" (pág. 1), con independencia de la dirección de la influencia (aunque la mayor parte de los casos se refieran a la influencia de la L1 en la L2). Justifican la elección del vocablo porque tiene un carácter bastante neutro, apropiado al extenso abanico de fenómenos que resultan del contacto entre las lenguas (transferencia, omisión, préstamo, etc.).

Por lo que se refiere al término transferencia, estos autores restringen su uso a "those processes that lead to the incorporation ${ }^{8}$ of elements from one language into another" (Kellerman y Sharwood Smith 1986:1). Con respecto a las palabras interferencia y "facilitación" (facilitation) y sus respectivas connotaciones negativas y positivas, afirman que aluden a procesos psicolingüísticos y que es preferible limitar su empleo al contexto de la teoría behaviorista.

Otros especialistas como Gass y Selinker (1983) apuntan que el término 'transferencia' es algo ambiguo ${ }^{9}$ En este sentido, sería necesario precisar en qué plano se va a analizar la influencia lingüística: en el plano del proceso que subyace a la actuación verbal o en el plano del producto resultante, ya sean errores o no. Según estos autores, sólo desde el punto de vista del producto, o resultado patente de la actuación del aprendiz, se muestra relevante la distinción entre transferencia positiva y negativa.

A pesar de la problemática terminológica y conceptual que rodea al fenómeno de la influencia entre lenguas, no cabe duda de que hay que adoptar algún vocablo/s para denominar el mencionado fenómeno. A este respecto hemos decidido utilizar el término transferencia para referir en sentido amplio la influencia y los efectos de la L1 sobre la L2, ya se manifiesten en forma de errores o no. La elección de este término viene motivada por ser uno de los más difundidos en la bibliografía específica consultada y porque en principio no implica ninguna valoración negativa. Somos conscientes, sin embargo, de sus limitaciones y de la complejidad del fenómeno en sus distintas manifestaciones, y coincidimos con Odlin (1989:28) en que:

A fully adequate definition of transfer seems unattainable without adequate definitions of many other terms, such as strategy, process, and simplification (...) Thus, one might plausibly argue that a fully adequate definition of transfer presupposes a fully adequate definition of language.

\section{Análisis diacrónico del papel de la transferencia linguiística}

Prácticamente desde que empieza a tener una cierta relevancia en el marco de la adquisición de una segunda lengua y hasta nuestros días, el fenómeno de la transferencia lingüística ha sido objeto de controversia. El presente apartado pretende ofrecer una visión diacrónica de las principales consideraciones sobre la influencia de la L1 en la adquisición

\footnotetext{
${ }^{8}$ Cursiva mía; nótese cómo emplean un término sin connotaciones negativas.

${ }^{9}$ Dechert y Raupach (1989:1 y ss.) recogen unas veinte definiciones diferentes de 'transferencia lingüística'.
} 
de la L2, contrastando para ello diferentes opiniones y teorías que se suceden en la segunda mitad del siglo $\mathrm{XX}^{10}$.

\section{Análisis Contrastivo}

Alrededor de los años cuarenta, la transferencia lingüística empieza a considerarse un factor importante dentro de la metodología y las teorías sobre la enseñanza y el aprendizaje de un segunda lengua. Estas teorías lingüísticas están muy influenciadas por la corriente psicológica dominante en la época: el behaviorismo (behaviorism), también denominado conductismo o conductivismo, que se encuadra dentro de las teorías ambientalistas del aprendizaje, según las cuales la educación o la experiencia y el entorno físico son más importantes para el desarrollo que la naturaleza o las dotes innatas ${ }^{11}$.

Para el behaviorismo, la adquisición lingüística es el resultado de la formación de hábitos, que se van creando por la asociación repetida y persistente de estímulos y respuestas, acompañadas éstas de refuerzos positivos ${ }^{12}$. Dentro de este enfoque, el papel de la L1 en la adquisición de la L2 se interpreta en términos de interferencia, es decir, los viejos hábitos de la L1 interfieren en el aprendizaje de los nuevos hábitos de la L2, pudiendo facilitar o dificultar la tarea:

We assume that the student who comes in contact with a foreign language will find some features of it quite easy and others extremely difficult. Those elements that are similar to his native language will be simple for him, and those elements that are different will be difficult (Lado 1957:2).

La convicción de que las diferencias entre la L1 y L2 dificultan el proceso de aprendizaje y son la causa de errores constituye el fundamento de la hipótesis del análisis contrastivo, según la cual se pueden predecir dichos errores basándose en estas diferencias. De esta forma, cuando dos lenguas son similares se producirá una transferencia positiva que facilitará el aprendizaje; cuando son diferentes se dará una transferencia negativa o interferencia que ocasionará áreas de dificultad y errores. Como resultado, se llevan a cabo numerosas comparaciones sistemáticas y contrastivas entre lenguas nativas y lenguas extranjeras ${ }^{13}$, estableciendo una jerarquía de dificultades de aprendizaje para pronosticar los posibles errores.

Pero a pesar de esta difusión de los estudios contrastivos, pronto empiezan a surgir diversas críticas provenientes de distintos autores. Por una parte, Chomsky ${ }^{14}$ desafía la

\footnotetext{
${ }^{10}$ Véase Odlin (1989), Ellis (1985) y Larsen-Freeman y Long (1994) para una visión panorámica del papel de la transferencia en la adquisición de una segunda lengua.

${ }^{11}$ Véase Larsen-Freeman y Long (1994:cap. 7.4).

${ }^{12}$ Véase B. F. Skinner, Verbal Behavior (New York 1957). Anteriormente L. Bloomfield, Language (New York 1933), ya había propuesto la idea del lenguaje como un eslabón en la cadena estímulo-respuesta.

${ }^{13}$ Véase Stockwell, Bowen y Martin (1965) para un análisis contrastivo inglés-español.

${ }^{14}$ Véase N. Chomsky, "Review of Verbal Behavior by B. F. Skinner", Language 35 (1959) 26-58.
} 
visión "behaviorista" de la adquisición del lenguaje; por otra, cuando las predicciones de los análisis contrastivos se someten a diversas pruebas empíricas, aparecen fallos importantes.

Con respecto a lo primero, Chomsky (1965) afirma que los seres humanos estamos dotados de manera innata (genéticamente) de un "dispositivo de adquisición del lenguaje" (language acquisition device) que contiene un conocimiento universal específico del mismo: la Gramática Universal. Según este autor, la adquisición lingüística no es el producto de la formación de hábitos por imitación y refuerzo (concepción behaviorista), sino de la formación de reglas. En este sentido, el "input" o entorno lingüístico al que está expuesto el niño actúa simplemente como un "detonador", que activa el bioprograma o herencia biológica que le permite inducir las reglas. Estas reglas son creativas y permiten a los aprendices generar y comprender expresiones nuevas y correctas.

Por extensión, estos planteamientos se aplican a la adquisición de la L2 $2^{15}$, y se empiezan a cuestionar las teorías del análisis contrastivo y la influencia de la L1 en dicho proceso de adquisición. A esto hay que añadir los resultados de distintas investigaciones empíricas que ponen en tela de juicio las predicciones del análisis contrastivo, ya que no es capaz de anticipar todos los errores y a veces los que son predichos no se dan luego en la práctica.

Ante esta pérdida de credibilidad de la hipótesis del análisis contrastivo, Wardhaugh (1970) propone distinguir entre una versión fuerte y una débil. La versión fuerte implica predecir los errores en el aprendizaje de una L2, basándose en un análisis contrastivo apriorístico de la lengua nativa y de la lengua extranjera, predicciones que no siempre se ven confirmadas. La versión débill, en cambio, empieza con los errores del aprendiz e intenta explicarlos, a posteriori, señalando las similitudes y diferencias entre las dos lenguas. Esta versión débil tiene mejor acogida y algunos análisis de errores recurren a ella en ciertos casos (véase apartado siguiente).

Análisis de errores

Al principio de la década de los setenta empiezan a realizarse numerosos análisis de errores con el objetivo de determinar sus tipos y causas. Entre las distintas razones que motivan la aparición de estas investigaciones hay que destacar fundamentalmente dos. De una parte, la imposibilidad del análisis contrastivo de explicar, y menos predecir, aquellos errores cometidos por los aprendices de una segunda lengua que no se deben a la influencia de la L1. De otra, la teorías de Chomsky y de la gramática generativa sobre la adquisición del lenguaje, que enfatizan los aspectos creativos de dicho proceso.

Como resultado, los errores empiezan a considerarse bajo una óptica diferente. Ya no se ven como el producto de la intrusión de hábitos de la L1, sino como resultado del intento

\footnotetext{
${ }^{15}$ Aunque las ideas de Chomsky han servido de base a numerosos autores para elaborar sus teorías (p. ej. Cook 1988; Krashen 1981), también han recibido críticas y se han cuestionado algunas de sus observaciones; p. ej. el hecho de que el aprendizaje finalice tan pronto o que ciertos principios sintácticos (dependencia de la estructura, subyacencia) no se puedan aprender y sean innatos (v. Larsen-Freeman y Long 1994:215-220).
} 
del aprendiz de generar y comprobar hipótesis. En este sentido, Corder (1967) considera los errores un reflejo de las distintas etapas del proceso creativo de adquisición de la L2. A él se debe la creación del término "dialecto idiosincrático" (idiosyncrasic dialect) ${ }^{16}$ para referirse a las características peculiares e individuales del habla del aprendiz.

Selinker (1972), por su parte, utiliza el término "interlengua" o "interlingua" (interlanguage), que define como "a separate linguistic system based on the observable output which results from a learner's attempted production of a TL [target language] norm" (pág. 214). Este sistema lingüístico, diferente de la L1 y de la L2, abarca una serie de estadios sucesivos y transitorios, que tienen que atravesar los aprendices durante el desarrollo de su competencia lingüística hasta llegar a la lengua meta. Según Selinker, la transferencia lingüística y las estrategias de aprendizaje, entre otros procesos, determinan la fosilización de ciertos elementos, es decir, unidades de la L1 que permanecen en la IL con independencia de la cantidad de instrucción.

Volviendo a las investigaciones basadas en los análisis de errores, éstas ponen de manifiesto que algunos se deben a la transferencia pero muchos otros son el resultado de fenómenos diferentes. A este respecto, Richards (1971) distingue entre errores de interlengua ${ }^{17}$ y errores de intralengua. Los primeros pueden atribuirse a la influencia de la L1 y normalmente los explica la versión débil de la hipótesis del análisis contrastivo; p. ej. 'Is the book of my friend': omisión del pronombre sujeto y expresión de la posesión con 'of' por influencia del español.

Los segundos, denominados por algunos autores errores del desarrollo (developmental errors), son los que cometen los aprendices con independencia de su L1 y no pueden explicarse por transferencia, sino más bien como resultado de las estrategias empleadas en la adquisición de la L2 ${ }^{18}$. Entre estas estrategias Odlin (1989:18-19) recopila las siguientes:

a) "Transferencia de instrucción" (transfer of training), derivada de la enseñanza recibida por el aprendiz; por ejemplo:

Profesor: Am I your teacher?

Estudiante: Yes, I am your teacher (Confusión de estructuras en un ejercicio de tipo mecánico)

b) "Sobregeneralización" (overgeneralization) : uso de una regla o estructura lingüística más allá de los límites de su campo de aplicación; p. ej. 'comed','goed' (extensión del morfema de pasado '-ed' de verbos regulares a verbos irregulares); 'mans','mouses' (uso del morfema de plural '-s' con sustantivos irregulares).

\footnotetext{
${ }^{16} \mathrm{~S}$. Corder, "Idiosyncrasic Dialects and Error Analysis", International Review of Applied Linguistics 5 (1971) 161-70.

${ }^{17}$ Nótese la diferencia entre este concepto de 'interlengua' y el de Selinker (1972).

${ }^{18}$ Ellis (1985:165) afirma que este tipo de estrategias no son específicas de los aprendices de una L2 sino que también recurren a ellas los nativos cuando adquieren la $\mathrm{L1}$, aunque los primeros las emplean con mayor frecuencia.
} 
c) "Simplificación" (simplification): omisión de diferentes elementos cuando su presencia es obligatoria; p. ej. 'I very good' (omisión de la forma verbal 'am'); 'Picture is very dark' (omisión del artículo).

Pero a pesar de las aportaciones del análisis de errores y sus taxonomías, este tipo de investigación no está exenta de críticas. Larsen-Freeman y Long (1994:64) mencionan que a veces es imposible encontrar un único origen a un error, o resulta difícil distinguir entre errores causados por transferencia y "errores del desarrollo" (p. ej. la omisión del sujeto en frases con el verbo 'to be' ocurre en el caso de niños españoles aprendices de inglés pero también aparece en el habla de los que adquieren inglés como L1).

Análisis de la actuación

La estrechez de perspectivas del análisis de errores lleva a algunos autores a incorporarlo al marco más amplio del denominado análisis de la actuación (LarsenFreeman y Long 1994:65 y ss.), en el que se incluyen los estudios de morfemas y el análisis de las secuencias del desarrollo.

\section{Estudios de morfemas}

Unos de los primeros estudios de morfemas ${ }^{19}$ en el ámbito de la adquisición de la L2 son los de Dulay y Burt $(1973,1974)$, que analizan el habla de distintos individuos para comprobar si emplean correctamente determinados morfemas en contextos obligatorios ${ }^{20}$. A raíz de estos trabajos y' otros posteriores, dichas autoras llegan a la conclusión de que existe un orden de adquisición de morfemas común para hablantes de diferentes lenguas maternas y por tanto independiente de la L1. Pero dentro de esta similitud Dulay, Burt y también Krashen reconocen una cierta variación, aunque mínima: "Individual learners show idiosyncratic behavior in certain aspects of language acquisition (...) Certain structures in English, such as articles, are particular susceptible to variation across subjects" (Dulay, Burt y Krashen 1982:202).

De los errores obtenidos sólo un porcentaje muy pequeño lo atribuyen a la transferencia de la L1 ("errores de interlengua"), rechazando así la hipótesis del análisis contrastivo. Estos últimos errores los explican por determinados factores del contexto o entorno (p. ej. presión del profesor en clase, necesidad de hacerse entender en un país extranjero y otros) que pueden llevar a un uso prematuro de la L2 cuando los hablantes todavía no están "mentalmente preparados" y no han adquirido las estructuras necesarias (Dulay, Burt y Krashen 1982:108-10). Por otra parte, rechazan la posibilidad de la transferencia positiva, basándose en ciertos errores que los aprendices no habrían cometido si hubiesen recurrido a las reglas de la L1.

\footnotetext{
${ }^{19}$ Entiéndase por 'morfema' la unidad mínima gramatical con valor distintivo pero que desempeña un papel menor en cuanto al significado de la oración: inflexiones nominales y verbales, artículos, auxiliares, preposiciones, etc.

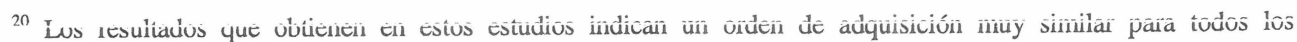
informantes, comenzando con el morfema de plural '-s' y terminando con el de la posesión con ' $s$ '.
} 
Según estos autores, aunque la influencia de la L1 del aprendiz es mínima en el terreno gramatical, sí deja una huella más significativa en la pronunciación, especialmente en el caso de adultos y niños principiantes. De todas formas la comunicación no se ve afectada en gran medida: "It is clear that communication is not seriously impeded by an accent, while an underdeveloped lexicon or lack of control over basic aspects of grammar precludes meaningful communication" (Dulay, Burt y Krashen 1982:112).

Los resultados obtenidos prueban, según este enfoque, la validez de la hipótesis de la construcción creativa (creative construction hypothesis) según la cual "universal cognitive mechanisms are the basis for the child's organization of a target language and it is the L2 system rather than the L1 system that guides the acquisition process" (Dulay y Burt 1974:52). Se trata, por tanto, de un proceso subconsciente en el que los niños, guiados por unos mecanismos mentales que parecen ser innatos (lo que estas autoras denominan el "organizador cognitivo" o cognitive organizer), organizan gradualmente el habla que oyen en función de una serie de reglas que construyen para generar frases. En este sentido el proceso de adquisición de la L2 utiliza medios muy similares a los que los niños emplean cuando adquieren su lengua materna, y esto queda reflejado en los errores del desarrollo similares en la adquisición de la L1 y de la L2 $2^{21}$.

Esta hipótesis abre un camino importante en la investigación al poner de manifiesto el papel de los mecanismos cognitivos o estrategias. Se va a criticar, en cambio, su intento de explicar la adquisición de la L2 prescindiendo por completo del papel de la $\mathrm{L1}^{22}$. A este respecto, la tónica general de la investigación actual es reconocer la influencia de la L1 como un factor más a tener en cuenta en la adquisición de la L2 (v. apdo. 2.4.).

Junto a los ya clásicos estudios de morfemas de Dulay y Burt, varios autores también realizan en los años setenta investigaciones de este tipo ${ }^{23}$ y obtienen resultados y conclusiones similares a los de las primeras. Algunos trabajos, en cambio, muestran ciertos aspectos que varían; por ejemplo, Larsen-Freeman (1976) recoge datos del habla de distintos aprendices de inglés (árabes, japoneses, persas y españoles) utilizando diferentes pruebas. Observa un orden de morfemas que coincide con el de Dulay y Burt pero sólo en los ejercicios de producción. Por otra parte, considera que la frecuencia del "input" o habla de los hablantes nativos influye en el orden de adquisición de morfemas en la L2. Existe, por tanto, una interacción entre los mecanismos cognitivos y ciertos factores del entorno lingüístico.

Pero al igual que ocurre primero con el análisis contrastivo y después con el análisis de errores, los estudios de morfemas reciben distintas críticas. Hatch (1983), por ejemplo, atribuye el hecho del pequeño porcentaje de errores debidos a la influencia de la L1 (especialmente en los estudios de Dulay y Burt) a ciertos problemas técnicos en la

\footnotetext{
${ }^{21}$ Véase Dulay, Burt y Krashen (1982:166-170) para una recopilación de ejemplos de este tipo de errores tomados de distintas fuentes.

${ }^{22}$ Véase Ellis (1985).

${ }^{23}$ Véase Hatch (1983:40-57) para una revisión de diferentes estudios de morfemas.
} 
interpretación de las estadísticas, así como a la propia prueba empleada ("Medición de la Sintaxis Bilingüe" o Bilingual Syntax Measure ${ }^{24}$ ). Según Hatch, esta técnica proporciona a veces resultados superficiales y un tanto contradictorios. Además, el número de morfemas empleado en los estudios constituye una mínima porción de la gramática inglesa. Por otra parte, los trabajos de Dulay y Burt se basan mayormente en niños, mientras que otros estudios han señalado que los adultos parecer estar más expuestos a la influencia de la L1 en comparación con los primeros ${ }^{25}$.

Liceras (1994:14), por su parte, considera que los estudios de morfemas en general "han mostrado un uso correcto en una etapa y no correcto en otra subsiguiente (...) [y] no está claro que algo que se produce correctamente en un momento dado se haya adquirido". Por eso, Ellis (1985) y otros autores prefieren hablar de orden de "precisión" (accuracy order) en lugar de orden de "adquisición".

Larsen-Freeman y Long (1994:86-90) recogen asimismo algunas de las criticas que se han hecho a los estudios de morfemas, pero acaban concluyendo con una valoración positiva:

A pesar de las limitaciones de algunos apartados, los estudios de morfemas proporcionan pruebas de que existen órdenes comunes de precisión / adquisición en las Ils [interlenguas]. En contra de lo que han afirmado algunos críticos ya hay, a nuestro modo de ver, demasiados estudios realizados con el suficiente rigor metodológico que muestran hallazgos generales lo bastante coherentes como para ignorar los elementos que tienen en común, de modo que algo debe haber detrás de todo esto (pág. 90).

Esta actitud positiva coincide en parte con la nueva valoración de los estudios de morfemas a partir de la década de los ochenta, abandonando algunos de sus objetivos iniciales y centrándose en el problema del papel de la instrucción formal en el orden de adquisición $^{26}$.

\section{Secuencias del desarrollo}

El estudio de las secuencias dell desarrollo (developmental sequences) también se incluye en el ámbito del "análisis de la actuación". Para algunos autores constituye una propuesta más esclarecedora que los 'estudios de morfemas' porque intenta descubrir cómo procesan los individuos el lenguaje en la adquisición, analizando los distintos pasos o etapas que atraviesan hasta llegar al dominio de una estructura particular.

\footnotetext{
${ }^{24}$ Esta procedimiento, también denominado en español "Modelo Bilingüe de Evolución Sintáctica" (Liceras 1992:105), consiste en una serie de viñetas en color que llevan al niño a participar con toda libertad en la conversación con el entrevistador.

${ }^{25}$ Véase Brown (1987).

${ }^{26}$ Ellis (1985:cap. IX) presenta un resumen de los resultados de distintos trabajos sobre este tema.
} 
Larsen-Freeman y Long (1994: 67) presentan un cuadro esquemático de las primeras investigaciones en este terreno. Así, por ejemplo, Dato ${ }^{27}$ compara la adquisición del sintagma verbal en español por hablantes nativos y por aprendices de inglés, y observa un modelo evolutivo parecido en ambos grupos. Basándose en los resultados obtenidos, estos primeros estudios llegan a la conclusión de que todos los aprendices de una L2, independientemente de la edad, la lengua nativa o el contexto del aprendizaje (formal o informal), parecen pasar por las mismas secuencias de desarrollo, y éstas son similares a las de los hablantes que adquieren una L1.

Pero hay que señalar igualmente que algunas investigaciones, aunque las menos al principio, reconocen ciertas diferencias entre ambas secuencias evolutivas. Wode ${ }^{28}$, por ejemplo, estudia la adquisición de la negación inglesa en hablantes nativos y en hablantes alemanes, y llega a la conclusión de que existen diferencias entre ambos, debido a que los niños alemanes confían en su L1 cuando hay una similitud básica entre ésta y la L2.

Como es de suponer, estos resultados contradictorios se han encontrado con opiniones diversas. Como muy bien apunta Ellis (1985:286), "Just how 'universal' the course of development is, however, remains a matter of some disagreement (...) It is not surprising that different researchers give different answers".

Autores como Dulay, Burt y Krashen (1982:122) consideran que existe una gran similitud entre las "construcciones de transición" (transitional constructions) ${ }^{29}$ en la adquisición de la $\mathrm{L} 1$ y de la $\mathrm{L} 2$ (hipótesis $\mathbf{L} \mathbf{1}=\mathbf{L} \mathbf{2})^{30}$, y estas semejanzas son producto de la construcción creativa. No obstante también observan algunas diferencias: "L2 learners appear to produce a wider variety of forms in one developmental phase than do L1 learners (e.g. this and these rather than just this in the beginning)". Y éstas, según ellos, se deben a factores relacionados con el "filtro afectivo" (affective filter) ${ }^{31}$, el entorno y la edad, entre otros, pero no dependen de la influencia de la lengua materna.

Ellis (1985) coincide en algunos aspectos con la teoría de los autores anteriores pero, a diferencia de éstos, reconoce la L1 como un factor a tener en cuenta en la adquisición de la L2. Dentro de este planteamiento, considera necesario distinguir entre secuencia de desarrollo y orden de desarrollo. La primera alude a ciertas etapas amplias que atraviesan

\footnotetext{
${ }^{27}$ D. Dato, "The Development of the Spanish Verb Phrase in Children's Second Language Learning", P. Pimsleur y T. Quinn (eds.), The Psychology of Second Language Learning. Papers from the Second International Congress of Applied Linguistics (London 1971) 19-33.

${ }^{28}$ H. Wode, "Developmental Sequences in Naturalistic L2 Acquisition", Working Papers on Bilingualism 11 (1976) $37-$ 57.

${ }^{29}$ Los mencionados autores utilizan este término para referirse a las 'secuencias del desarrollo'.

${ }^{30}$ Esta hipótesis postula que la adquisición de la L2 no difiere esencialmente de la adquisición de la L1 y es independiente de cualquier L1.

31 Estos autores entienden por "filtro afectivo" una parte del sistema de procesamiento interno que filtra inconscientemente lo que los aprendices oyen, en función de la motivación, las necesidades, las actitudes y el estado emocional de los mismos, afectando al ritmo y a la calidad del aprendizaje.
} 
todos los aprendices de la L2. En este sentido es "universal" y producto del proceso de construcción creativa. Con respecto a la similitud entre las secuencias del desarrollo de la L1 y la L2, este autor señala una semejanza de procesos hasta cierto punto aunque no una identidad entre ambos tipos de adquisición: "Certainly the L1=L2 acquisition hypothesis has not been proved in its strong form (...) In SLA [second language acquisition] both the L1 and some factors to do with the learner's more advanced cognitive development play a part" (Ellis 1985:67).

El 'orden de desarrollo', por su parte, se refiere al orden en que se adquieren en la L2 ciertos rasgos gramaticales específicos y está sujeto a variación ${ }^{32}$. Este autor explica muy bien la relación entre ambos conceptos con una metáfora: "learners take the same road but do not necessarily drive along it in the same way. They follow a standard sequence but vary in the order in which specific features are acquired" (Ellis 1985:64). Estas diferencias se derivan de la L1 del aprendiz, de factores lingüísticos y extralingüísticos del entorno (variación en el "input", grado de integración social en la comunidad de la lengua meta, etc.) y de otros relacionados con el estilo o las preferencias individuales.

En cuestiones como ésta que nos ocupa es difícil encontrar una opinión generalizada y unitaria. No obstante, puede decirse que en la actualidad las investigaciones en general se acercan más en algunos aspectos a la posición de Ellis que a las de Dulay, Burt y Krashen. En concreto, nos referimos a la consideración de que la influencia de la L1 no altera las secuencias normales de los procesos de construcción creativa, pero puede modificar su paso por ellas, retrasando o acelerando el comienzo de alguna secuencia en función de la mayor o menor semejanza de la forma de desarrollo con la estructura de la $\mathrm{L} 1^{33}$.

\section{Revalorización del papel de la transferencia. Consideraciones actuales}

Hoy en día, la opinión más avalada por los estudios sobre adquisición de una segunda lengua tiende a reconocer, en mayor o menor grado, el papel de la L1 como un factor más en dicho proceso de adquisición. En el presente apartado se van a considerar algunos de los planteamientos más significativos. Si bien es cierto que es difícil encontrar una opinión uniforme en este campo de la investigación, no existen claros defensores de una adquisición de la L2, especialmente para adultos, que prescinda por completo de la capacidad cognitiva que supone la L1. Por otra parte, aunque la mayoría de los estudiosos reconocen la influencia de la lengua materna, también admiten que la dificultad principal de esta cuestión estriba en precisar su papel y delimitar su campo de actuación:

Es evidente que casi todo lo que sabemos sobre la transferencia lo hemos aprendido en la última década; es igualmente claro lo mucho que falta por aprender para poder predecir con cierta seguridad cuándo y cómo se producirá la transferencia de la L1 (Larsen-Freeman y Long 1994:105).

\footnotetext{
${ }^{32} \mathrm{Cfr}$. los estudios de morfemas, que postulan un orden de adquisición común para los distintos aprendices.

${ }^{33}$ Véase Larsen-Freeman y Long (1994:105).
} 
Varied aspects of L2 learning need to be investigated before it can be decided how and when the first language is involved in the learning of the second. Though transfer from the L1 indeed turns out to be important, often in unexpected ways, its role needs to be established through properly balanced research (...) (Cook 1991:6).

\section{La LI y la Gramática Universal}

En la mayoría de los trabajos recientes prevalece la idea de la adquisición de una lengua como resultado de la formación de reglas o del contexto paramétrico, aunque también influyen otros factores. En el caso concreto de la adquisición de la L2, Ellis (1985), Cook (1991), Larsen-Freeman y Long (1994) y otros reconocen varios aspectos implicados en la misma: la Gramática Universal con los principios y parámetros ${ }^{34}$, los factores lingüísticos (formales y pragmáticos) específicos de la L1 y de la L2, y una serie de factores psicolingüísticos y sociolingüísticos ${ }^{35}$.

En relación con lo anterior, Liceras(1992:25) considera que la L1 juega un papel de intermediaria en la fijación de parámetros de la L2, es decir, tiene un papel de mediadora entre la Gramática Universal y los datos de la L2. White (1986) es partidaria de una interpretación similar y afirma que los aprendices, a la hora de fijar los parámetros para la L2, comienzan a menudo por su L1. En su estudio compara la adquisición del inglés ("nonpro-drop") por hablantes de español ("pro-drop") ${ }^{36}$. Los nativos españoles asumen en un principio que la expresión del sujeto en inglés, como ocurre en español, no es obligatoria, pero luego aprenden que sí lo es.

\section{Transferencia y similitud L1/L2}

El reconocimiento de la influencia de la L1 en la adquisición de la L2 guarda relación, según Ellis (1985:33), con una evolución de la hipótesis del análisis contrastivo, que ahora presenta la transferencia bajo una óptica diferente. A este respecto señala Liceras (1992:25) que:

El rechazo indiscriminado de la influencia de la L1, que se explicaba por la necesidad de separarse de las teorías conductistas del aprendizaje que habían hecho de la interferencia la clave para explicar la IL [interlengua], ya no se siente como una necesidad y, por tanto, la L1 vuelve a ocupar un papel central en la teoría de la adquisición de L2.

\footnotetext{
${ }^{34}$ Según Chomsky (1981) la Gramática Universal se compone de principios y parámetros. Los primeros son innatos y abstractos, están presentes en la mente de todos los hablantes y determinan qué combinaciones son posibles en las lenguas humanas. Los parámetros son aspectos que varían de una lengua a otra dentro de unos límites muy precisos. Véase también Cook (1988).

${ }^{35}$ Éstos factores extralingüísticos incluyen aspectos varios como la personalidad (motivación, ansiedad, empatía, etc.), el tipo de aprendiz, los estilos de aprendizaje, el nivel de competencia, la integración social, etc.

${ }^{36}$ El parámetro "pro-drop" está relacionado con la omisión del sujeto. Las lenguas "pro-drop" (p. ej. español) pueden omitirlo, mientras que en las lenguas "non-pro-drop" (p. ej. inglés, francés) su presencia es obligatoria.
} 
Una de los avances de la mencionada hipótesis es reconocer que existe una mayor probabilidad de transferencia cuando hay una cierta similitud entre las estructuras de la L1 y de la L2 en lugar de diferencias: "The contrastive analysis hypothesis might, however, be reinstated in the form that absence of perceived similarities, for instance between totally unrelated languages, produces learning conditions where transfer does not easily develop" (Ringbom 1990:216).

Ringbom (1986) realiza un estudio sobre el aprendizaje del inglés en hablantes de finlandés y hablantes de sueco (el sueco es más parecido estructuralmente al inglés que el finlandés), y llega a la conclusión de que la semejanza entre la L1 y la L2 tiene una influencia positiva y facilita el proceso de aprendizaje: "(...) in the learning of a closely related L2, the learner's L1 has already provided him with a considerable part of this essential knowledge automatised in his mind" (Ringbom 1986:159). No obstante, reconoce que estos efectos son más palpables en la comprensión que en la producción ${ }^{37}$. Por otra parte, este autor especifica en un trabajo posterior (Ringbom 1990:216) que esta facilitación es más evidente en el caso de las estructuras simples, características de las primeras etapas del proceso de aprendizaje:

The basic facilitating effect of L1-transfer is no longer obvious when the learner starts learning more complex relationships than the simplified one-to-one equivalences between L1 and L2 that he or she established at the beginning and soon has to modify.

Otra de las modificaciones de la hipótesis del análisis contrastivo es reconocer que la transferencia de la L1 también puede manifestarse en forma de estrategia de 'omisión' o 'evasión' (avoidance). En este sentido, cuando los aprendices perciben que determinadas estructuras o funciones de la lengua meta son bastante diferentes y/o dificiles con respecto a las correspondientes de la lengua nativa, pueden evitar su empleo en lugar de cometer errores $^{38}$.

\section{Transferencia como estrategia comunicativa}

Otra de las consideraciones que más ha contribuido a la revalorización de la transferencia ha sido su interpretación en un marco más acorde con el enfoque mentalista, que tiene en consideración la contribución activa del individuo en el proceso de aprendizaje. En este contexto, Corder $^{39}$ reinterpreta el concepto de interferencia como

${ }^{37}$ Cfr. W. F. Mackey, Language Teaching Analysis (London 1965) 109: "If [a learner] (...) is learming simply to understand the language, the greater the similarity between the first language and the second, the easier the latter will be to understand. In using the language, however, it is the similarity that may cause interference by the misuse of such things as deceptive cognates".

${ }^{38}$ Cfr. H. Kleinmann, "Avoidance Behavior in Adult Second Language Acquisition", Language Learning 27 (1977) 93 107. Este autor apoya el mencionado planteamiento, pero sugiere que ciertos aspectos de la personalidad como la ansiedad y el temor a cometer errores influyen igualmente en la omisión de estructuras.

${ }^{39} \mathrm{~S}$. Corder, "Language Distance and the Magnitude of the Language Learning Task", Studies in Second Language Acquisition 2 (1978) 27-36. 
"intercesión" (intercession), una estrategia comunicativa a la que puede recurrir el aprendiz cuando quiere expresarse en la L2 pero no tiene los recursos lingüísticos necesarios ${ }^{40}$. La siguiente cita de Brown (1987:54) recoge igualmente esta idea:

The first language (...) may be more readily used to bridge gaps that the adult learner cannot fill by generalization within the second language. In this case we do well to remember that the first language can be a facilitating factor, and not just an interfering factor.

Faerch and Kasper (1984), por su parte, señalan que dentro de las estrategias comunicativas que intentan resolver un problema (achievement strategies), hay que distinguir entre las que se apoyan en la L1 y las de la L2. El primer tipo, que es el que nos atañe, incluye a su vez las siguientes:

- Cambio de código (code-switching): el aprendiz utiliza una forma de la L1 en la L2; p. ej. 'I don't have any Geschwester (alemán)'; 'I must go to the farmacia'.

- Préstamo (foreignizing): se emplea una forma de la L1 pero se adapta para que parezca una forma de la L2; p. ej. 'papirkurv' (danés) > 'papercurve'; sufijo '-tion' para formar palabras inglesas a partir de las españolas: 'manifestación' > 'manifestation'

- Calco o traducción literal; p. ej. 'newspaper' > 'noticias de papel' ('periódico'); 'bedroom' > 'habitación de cama' ('dormitorio').

\section{Conclusiones}

A lo largo del presente trabajo se han descrito distintos planteamientos teóricos que muestran el papel cambiante que ha desempeñado la transferencia, o influencia de la L1 en la adquisición de la L2, en la segunda mitad del siglo XX. Tras la consideración negativa de la transferencia entendida como 'interferencia' por el análisis contrastivo, y el papel mínimo que le atribuyen los primeros trabajos sobre análisis de errores, estudios de morfemas y secuencias del desarrollo, ha pasado a tener una valoración más equilibrada.

Hoy se acepta mayoritariamente que la L1, como mecanismo linguo-cognitivo, es un factor a considerar en la adquisición de la L2, aunque ya resulta más difícil precisar la importancia de la misma, así como las causas que la motivan. No siempre se puede determinar si la transferencia es el origen de ciertas construcciones o procesos, y a veces resulta más productiva en determinados aprendices (variación individual) y lenguas. En este sentido hay que tener cuidado con las generalizaciones excesivas sobre el papel de la lengua materna, ya que en el proceso de adquisición de la L2 la transferencia interacciona con otros factores lingüísticos y extralingüísticos de forma compleja, siendo necesaria una investigación más exhaustiva sobre esta cuestión. Como indica Odlin (1989:41), "(...) the

\footnotetext{
${ }^{40}$ Cfr. E. Kellerman, "If at First you Do Succeed...", S. Gass y C. Madden (eds.), Input in Second Language Acquisition (Rowley, Mass. 1983) 345-353. Este autor analiza la relación entre el uso de la lengua materna y el nivel de competencia de los aprendices en la segunda lengua.
} 
relation between transfer and other processes in second language acquisition remains only partially understood".

\section{Bibliografía}

Brown, H. D. 1987. Principles of Language Learning and Teaching. $2^{\mathrm{a}}$ ed. Englewood Cliffs, N. J.: Prentice-Hall.

Cook, V. J. 1988. Chomsky's Universal Grammar: An Introduction. Oxford: Blackwell.

Cook, V. J. 1991. Second Language Learning and Language Teaching. London: Edward Arnold.

Corder, S. P. 1967. "The Significance of Learner's Errors", IRAL 4, 161-170.

Chomsky, N. 1965. Aspects of the Theory of Syntax. Cambridge, Mass.: MIT Press.

Chomsky, N. 1981. "Principles and Parameters in Syntactic Theory", N. Hornstein y D. Lightfoot (eds.). Explanations in Linguistics: The Logical Problem of Language Acquisition. London: Longman.

Dechert, H., M. Brüggemeir y D. Fütterer (comps.). 1984. Transfer and Interference in Language: A Selected Bibliography. Amsterdam: John Benjamins.

Dechert, H. W. y M. Raupach (eds.). 1989. Transfer in Language Production. Norwood, N. J.: Ablex.

Dulay, H. y M. Burt. 1973. "Should we Teach Children Syntax?", Language Learning 23.2, 245-258.

Dulay, H. y M. Burt. 1974. "Natural Sequences in Child Second Language Acquisition", Language Learning 24, 37-53.

Dulay, H., M. Burt y S. Krashen. 1982. Language Two. New York: Oxford University Press.

Ellis, R. 1985. Understanding Second Language Acquisition. Oxford: Oxford University Press.

Faerch, C. y G. Kasper. 1984. "Two Ways of Defining Communication Strategies", Language Learning 34, 45-63.

Gass, S. y L. Selinker (eds.). 1983. Language Tansfer in Language Learning. Rowley, Mass.: Newbury House.

Hatch, E. 1983. "Morphology”, Psycholinguistics: A Second Language Perspective. Rowley, Mass.: Newbury House, 40-57.

Kellerman, E. y M. Sharwood Smith. 1986. "Crosslinguistic Influence in Second Language Acquisition: An Introduction", E. Kellerman y M. Sharwood Smith (eds.). Crosslinguistic Influence in Second Language Acquisition. New York: Pergamon Press, 1-9. 
Krashen, S. 1981. Second Language Acquisition and Second Language Learning. Oxford: Pergamon Press.

Lado, R. 1957. Linguistics Across Cultures. Ann Arbor, Michigan: The University of Michigan Press.

Larsen-Freeman, D. 1976. "An Explanation for the Morpheme Acquisition Order of Second Language Learner”, Language Learning 26.1, 125-134.

Larsen-Freeman, D. y M. H. Long. 1994. Introducción al estudio de la adquisición de segundas lenguas. Madrid: Gredos. Trads. I. Molina Martos y P. Benítez Pérez de An Introduction to Second Language Acquisition Research. London: Longman, 1991.

Liceras, J. M. 1992. "Hacia un modelo de análisis de la interlengua" en J. M. Liceras (comp.). La adquisición de las lenguas extranjeras: Hacia un modelo de análisis de la interlengua. Trad. M. Marcos. Madrid: Visor Dis, 11-27.

Odlin, T. 1989. Language Transfer: Cross-linguistic Influence in Language Learning. Cambridge: Cambridge University Press.

Richards, J. 1971. "Error Analysis and Second Language Strategies", Language Sciences 17, $12-22$.

Ringbom, H. 1986. “Crosslinguistic Influence and the Foreign Language Learning Process”, E. Kellerman y M. Sharwood Smith (eds.). Crosslinguistic Influence in Second Language Acquisition. New York: Pergamon Press, 150-162.

Ringbom, H. 1990. "Effects of Transfer in Foreign Language Learning", H. W. Dechert (ed.). Current Trends in European Second Language Acquisition. Clevedon: Multilingual Matters, 205-218.

Selinker, L. 1972. "Interlanguage”, IRAL 10.2, 209-231.

Stockwell, R. P., J. D. Bowen y J. W. Martin. 1965. The Grammatical Structures of English and Spanish. Chicago: University of Chicago Press.

Wardhaugh, R. 1970. “The Contrastive Analysis Hypothesis”, TESOL Quarterly 4, 123-130.

Weinreich, U. 1953. Languages in Contact: Findings and Problems. The Hague: Mouton.

White, L. 1986. "Implications of Parametric Variation for Adult Second Language Acquisition: An Investigation of the Pro-Drop Parameter", V. J. Cook (ed.). Experimental Approaches to Second Language Learning. New York: Pergamon Press, 55-72. 